\title{
Robust Monetary Policy under Model Uncertainty and Inflation Persistence
}

\author{
Li Qin*, Moïse Sidiropoulos**, Eleftherios Spyromitros* \\ * University Louis Pasteur of Strasbourg (France), BETA-Theme, \\ ** Aristotle University of Thessaloniki (Greece) and BETA-Theme,
}

15th September 2008

\begin{abstract}
This paper examines the relationship between the preference for robustness of central bank (when it fears that its model is misspecified), the inflation persistence and the output cost of disinflation. Using a simple monetary game model with misspecification, this paper shows that higher preference for robustness of central bank is positively associated with the inflation persistence and thus negatively with the speed of disinflation.
\end{abstract}

Keywords : Model uncertainty, Robust control. Minmax policies, Inflation persistence, Sacrifice ratio

JEL classification : E50, E52, E58

Over the last decade, there is a rapidly growing literature to explain the high degree of inflation persistence observed in the data. Recently, a research network of economists from the national central banks of the euro area and the European Central Bank (ECB) have been investigating the empirical evidence for inflation persistence, its determinants and implications for monetary policy (see Angeloni et al., 2004 for a summary), and alternative interpretations are proposed to explain and remedy the 
high inflation persistence found in post-war U.S. data (see Taylor, 2000 and Cogley and Sargent, 2001).

In this context, several authors (Fuhrer and Moore, 1995; Fuhrer, 2000; Calvo et al., 2001; Christiano et al., 2005; Blanchard and Gali, 2007) have proposed different mechanisms to build inflation persistence into the deep structure of the economy. Another point of view suggests that the degree of inflation persistence is not only an inherent structural characteristic of industrial economies, but rather may be due to changes in the orientation of monetary policy (Sargent, 1999; Taylor, 2000, Goodfriend and King, 2001; Westelius, 2005). Thus, in an environment with independence of central bank and transparency of monetary policy, inflation expectations may become contained and, hence, price and wage setters may be less inclined to change their contracts in response to shocks. In this respect, the way economic agents perceive major changes in the policy regime, such as central bank independence and transparency of monetary policy, has important implications for the short-run impact of monetary policy changes. In effect, the speed at which the economy reacts to a change in its environment may be slow and the economy may not move quickly to a new equilibrium with lower price expectations.

On the other hand, the degree of inflation persistence represents a key parameter of the monetary transmission mechanism and thus, it has important implications for the ability of monetary policy to stabilise inflation relative to output as well as a significant impact on the output cost of disinflation or sacrifice ratio (Diana and Sidiropoulos, 2004). In effect, the question of how monetary policy should be set optimally when the structure of the economy exhibits inflation persistence is crucial for monetary policy authorities. However, the above literature is focused on how the characteristics of the central banks may influence the inflation persistence without taking into account the fact that policymakers may not have a complete knowledge of the true structure of the economy. It involves thus the issue of model uncertainty. Taking account of model uncertainty may shed new light on the ongoing controversy about the appropriate specification of inflation persistence.

Without the possibility to have a complete description of reality, a policymaker is likely to prefer basing policy on principles that are valid also if the assumptions on which 
the model is founded differ from reality. Actually, models rest on a set of assumptions that may or may not be good approximations of true economies. In other words, policy prescriptions should be robust to reasonable deviations from the benchmark model. The growing literature on monetary policy robustness has been developed via three directions. The first one leads to what has been called robustly optimal instrument rules (Svensson and Woodford, 2004; Giannoni and Woodford, 2003a, 2003b). As these instrument rules do not depend on the specification of the generating processes of exogenous disturbances in the model, they are, therefore, robust to misspecification in these processes. The second one, initiated by Hansen and Sargent (2001, 2007), corresponds to robust control approach to the decision problem of agents who face model uncertainty. In the sense of Hansen and Sargent, robust monetary policies are designed to perform well in worst-case scenarios. These policies arise as the equilibrium in a game between the monetary authorities and an evil agent who chooses model misspecification to make the authorities look as bad as possible. The third approach to robust control is called structured Knightian uncertainty where the uncertainty is assumed to be located in one or more specific parameters of the model, but where the true values of these parameters are known only to be bounded between minimum and maximum conceivable values (Onatski and Stock 2002; Giannoni, 2002, 2007; Tetlow and von zur Muehlen, 2004).

The objective of our paper is to examine analytically the effects of increased model uncertainty, in the sense of Hansen and Sargent (2007), on the inflation persistence and the sacrifice ratio using a simple Barro-Gordon economy model and where inflation persistence is introduced through the data generating process for the structural shocks hitting the economy.

The paper is structured as follows. Section 2 presents the model. Section 3 introduces the robust monetary policy. Section 4 analyzes the relationship between inflation persistence and the preference for robustness and the effects of robustness on the sacrifice ratio. Section 5 concludes. 


\section{The model}

We consider a simple Barro-Gordon (1983) monetary game model extended to allow for persistent stochastic supply-side shocks (Rogoff, 1985) and indexed wage contracts (Ball, 1998; Gray, 1976). Output is given by using a Cobb-Douglas production function to transform the sole variable input, homogenous labour, in combination with other, fixed, factors of production. Thus, the relationship between output and employment at the aggregate level is described by the following log-linear production function:

$$
y_{t}=\alpha l_{t}+u_{t}, \quad 0<a<1
$$

where $y_{t}$ is the log of output, $l_{t}$ is the log of employment and the parameter $u_{t}$ represents a random supply-side shock to production technology (to be specified below). Firms decide on labour demand, $l_{t}^{d}$, and output by maximizing their profits, i.e.,

$$
l_{t}^{d}=\ln \arg \max _{L_{t}}\left\{P_{t} Y_{t}-W_{t} L_{t} \mid Y_{t}=L_{t}^{\alpha} \cdot \exp \left(u_{t}\right)\right\}
$$

where capital letters denote the according non-logarithmic variables. Thus, by equalizing the marginal product of labour to the real wage, the labour demand function is

$$
l_{t}^{d}=\tilde{l}-\frac{1}{1-\alpha}\left(w_{t}-p_{t}-u_{t}\right), \quad \tilde{l}>0
$$

where $\tilde{l}=\ln (\alpha) /(1-\alpha), w_{t}$ is the $\log$ of nominal wage and $p_{t}$ the log of output price in time $t$. All workers are members of the economy-wide union, with the available supply labour, $l_{t}^{s}$, given by:

$$
l_{t}^{s}=\tilde{l}-\delta+\eta\left(w_{t}-p_{t}\right), \quad \delta>0, \quad \eta \geqslant 0
$$

where the intercept term in (4) is not set equal to that of the demand for labour because we assume that the labour supply is affected by distortions in the labour market, captured by the parameter $\delta$. Equating (3) and (4), and assuming, without any loss of generality, that $\eta=0$ (i.e., desired supply of labor is assumed to be completely inelastic), we obtain:

$$
\widehat{w}_{t}=p_{t}+\delta(1-\alpha)
$$


where $\widehat{w}_{t}$ is the market-clearing or competitive equilibrium nominal wage that would arise in the absence of nominal wage contracts and leads to the following competitive equilibrium output level : $\widehat{y}_{t}=\tilde{y}-\kappa+u_{t}$, with $\tilde{y}=\alpha \tilde{l}$ and $\kappa=\alpha \delta$.

The treatment of wage determination, like that of Ball (1988), follows Gray (1976). Wage contracts are negotiated and signed at the beginning of each period, prior to the observation of the disturbances. These contracts specify a base wage, $E_{t-1} \widehat{w}_{t}$, set at the expected market-clearing value, i.e., such that $E_{t-1} l_{t}^{d}=l_{t}^{s}$, and an indexation parameter, $\gamma$, relating the actual nominal wage to unexpected movements in the price level, $\left(p_{t}-E_{t-1} p_{t}\right)$, following the indexing rule:

$$
w_{t}=E_{t-1} \widehat{w}_{t}+\gamma\left(p_{t}-E_{t-1} p_{t}\right), \quad 0 \leq \gamma \leq 1
$$

where $E_{t-1}$ is the rational expectation operator and $\gamma$ is the indexing parameter. For $\gamma=1$, wages are fully indexed, for $0<\gamma<1$, wages are partially indexed and for $\gamma=0$, there is no indexation. Thus, a moral hazard problem arise, justifying the incentive of workers to index their nominal wages to unexpected price movements.

Once contracts are signed, workers are committed to supplying whatever amount of labor firms demand, and employment, $l_{t}$, is purely demand determined. The output is determined by the level of employment since capital is assumed fixed. Integrating thus the equation (6) into (3) and using (1), we obtain the following aggregate output supply function:

$$
y_{t}=(\tilde{y}-\kappa)+\xi(1-\gamma)\left(\pi_{t}-E_{t-1} \pi_{t}\right)+(1+\xi) u_{t}, \quad \xi=\alpha /(1-\alpha)>0
$$

where, $\pi_{t}\left(=p_{t}-p_{t-1}\right)$ is the inflation rate and $E_{t-1} \pi_{t-1}\left(=E_{t-1} p_{t}-p_{t-1}\right)$ is the expected inflation rate. Finally, the parameter $u_{t}$, representing a random supply-side shock in this model, is assumed to pursue the following process:

$$
u_{t}=\phi u_{t-1}+\epsilon_{t}, \quad 0 \leq \phi \leq 1
$$

where $\phi$ is the degree of autocorrelation in random supply-side shocks and $\epsilon_{t}$ is a normally distributed random variable with zero mean and a variance varying with $\phi$ so as to standardize the variance of $u_{t}$ at $\sigma_{u}^{2}$, i.e., $\epsilon_{t} \sim N\left[0,\left(1-\phi^{2}\right) \sigma_{u}^{2} I\right]$. Note that the 
specification of the way in which supply-side shocks evolve over time in (8) is motivated by the choice of a simpler way to introduce the inflation persistence in this model (see Bleaney, 2001; Diana and Sidiropoulos, 2004) than through models including the inertia that overlapping wage contracts impart to the inflation rate (Taylor, 1980). In this context, the autocorrelation coefficient $\phi$ shows that a random productivity shock is persistent and, since $u_{t}$ depends on $u_{t-1}$, this shock will be transmitted forward in time generating thus an inflation persistence.

\section{Robust monetary policy}

To design the robust monetary policy, the central bank takes into account a certain degree of model misspecification by minimizing its objective function in the worst possible model within a given set of plausible models.

\section{$2.1 \quad$ Introducing misspecification}

We clarify here the monetary policy by assuming that the central bank sets the inflation rate, $\pi_{t}$, to minimize a standard objective function that is quadratic in deviations of the output and inflation from their targets levels :

$$
\min _{\left\{\pi_{t}\right\}} \mathrm{E}_{0 t=0}^{\infty} \beta^{t}\left[\left(y_{t}-y^{*}\right)^{2}+\lambda \pi_{t}^{2}\right], \quad \lambda>0
$$

where $y^{*}$ and $\pi^{*}$ are respectively the output and inflation targets, with $\pi^{*}$ normalized to zero without any loss of generality. The parameter $\lambda$ denotes the central bank's weight on inflation stabilization relative to the output stabilization and the output target, $y^{*}$ $=\widehat{y}_{t}+\kappa$, is expressed in percentage points above the natural output, $\widehat{y}_{t}$. However, the central bank has an uncertainty about model misspecification. Even if the model (7) is seen as the most likely model, the central bank admits that this reference model may be misspecified. For that reason, it requests to design its monetary policy to be robust against deviations from the reference model. To formalize these uncertainties about model misspecification, we follow Hansen and Sargent (2007) and introduce in equation (7) a specification error, designated by $v_{t}$. Thus, the misspecified model is given by 


$$
y_{t}=(\tilde{y}-\kappa)+\xi(1-\gamma)\left(\pi_{t}-E_{t-1} \pi_{t}\right)+\phi(1+\xi) u_{t-1}+\epsilon_{t}+v_{t}
$$

The two disturbances terms and have different properties. The term $u_{t}$ is assumed to be a random error with a prior known stochastic properties, whilst $v_{t}$ represents in the spirit of robust control a totally ambiguous model misspecification error, in the sense that the policymaker is not able to assign any prior probability distribution to $v_{t}$. The model with $v_{t}=0$ represents the reference model, while the models with $v_{t} \neq 0$ represent candidate models surrounding the reference model. In this context, as the central bank is assumed to be unable to provide a probability distribution over different deviations from the reference model, it instead designs its monetary policy to be optimal in the worst possible outcome within a neighbourhood of reference model. Hence, the central bank's doubts for misspecification may be formalized by assuming that the worst-case specification errors are chosen by a fictitious evil agent to maximize central bank loss subject to some constraints specified below.

Thus, the worst-case model is the model in which the central bank selects the inflation rate to minimize its loss function while the evil agent selects the specification errors to maximize loss. This is the outcome that the central bank worries the most and against which it desires monetary policy to be robust. On the other hand, a more likely outcome of the model is one where the central bank sets policy and agents form expectations to reflect misspecification in the worst-case model. However, when there is no such misspecification, the reference model turns out to be correct.

\section{$2.2 \quad$ Setting up the control problem}

In this context, the central bank allocates, according to its preference for robustness, a budget, $h$, to the evil agent, that is used to create misspecification in equation (7). The standard robust control problem would have a common budget constraint on misspecification in all equations of the model. This budget constraint is then given by

$$
\mathrm{E}_{0 t=0}^{\infty} \beta^{t}\left(v_{t}\right)^{2} \leq h_{t}^{2}
$$


where the parameter $h_{t}$ bounds the square of the central banks specification error $v_{t}^{2}$. Thus, the size of the distortion term $v_{t}$ must be bounded as the central bank's reference model remains an approximation of the real world system. Following Hansen and Sargent (2007), the robust monetary policy is obtained by solving the minmax problem

$$
\min _{\left\{\pi_{t}\right\}} \max _{\left\{h_{t}\right\}} \mathrm{E}_{0}^{\infty} \beta_{t=0} \beta^{t}\left[\left(y_{t}-y^{*}\right)^{2}+\lambda \pi_{t}^{2}-\theta v_{t}^{2}\right],
$$

subject to the misspecified model (10) end the evil agent's budget constraint in (11). The central bank thus sets the inflation rate to minimize the value of its loss function, while the evil agent sets its controls to maximize the central bank's loss. The Lagrangian for this problem is given by

$$
\begin{aligned}
\mathrm{E}_{0} \underset{t=0}{\infty} \beta^{t} & {\left[\left(y_{t}-y^{*}\right)^{2}+\lambda \pi_{t}^{2}-\theta v_{t}^{2}\right] } \\
& \quad-\mu_{t}\left[y_{t}-(\tilde{y}-\kappa)-\xi(1-\gamma)\left(\pi_{t}-E_{t-1} \pi_{t}\right)-\phi(1+\xi) u_{t-1}-\epsilon_{t}-v_{t}\right]
\end{aligned}
$$

where the $\mu_{t}$ is the Langrange multiplier on the constraint (10) and the parameter $\theta$ desires to be robust. This parameter is related to the evil agents budget $h$, and determines the set of models available to the evil agent against which the policymaker indicates the degree of model uncertainty, as well as the central bank's preference for robustness. As $h$ approaches zero, the parameter $\theta$ approaches to infinity $(\theta \rightarrow \infty)$, and the degree of misspecification approaches zero. This represents the case without model uncertainty. Inversely, a smaller value of $\theta$ means an increasing degree of model uncertainty inducing greater preference for robustness.

\subsection{Optimality conditions}

Assuming that neither the central bank nor the evil agent has access to any commitment mechanism, we take expectations as given in the optimization and look for a discretionary equilibrium. From the first-order conditions we derive the following optimality conditions relating output, $y_{t}$, inflation, $\pi_{t}$, and the degree of misspecification, $v_{t}$, to each other: 


$$
\begin{aligned}
y_{t}-y^{*} & =-\left[\frac{\lambda}{(1-\gamma) \xi}\right] \pi_{t} \\
v_{t} & =\frac{1}{\theta}\left(y_{t}-y^{*}\right)
\end{aligned}
$$

Combining equations (14) and (15) we get

$$
\pi_{t}=-\frac{\theta}{\lambda} \xi(1-\gamma) v_{t}
$$

An interesting implication of these results is that the optimal inflation-output tradeoff in equation (14) is not affected by the presence of model uncertainty illustrated by the central bank's preference for robustness $\theta$ (see Walsh, 2004). On the other hand, equation (14) shows that the optimal monetary policy leans against the wind, reducing the output when inflation is high. The coefficient $[\lambda /(1-\gamma) \xi]$ of the optimal trade-off illustrates that if the central bank assigns a large weight on inflation stabilization $(\lambda)$ or if monetary policy has stronger effects on inflation through the output $(\gamma$ is large and $\xi$ is small), the optimal trade-off is steeper, so the central bank reduces output more when inflation is high.

We consider also that the worst-case specification error $v_{t}$ in equation (16) is larger in absolute value when inflation is far away from steady state. This error tends to push inflation even further away, through specification error in the aggregate output supply function (10). This specification error forces the central bank to move the output further to achieve the desired trade-off between inflation and the output [see equation (14)]. When the output $y_{t}$ is below its target $y^{*}$, the misspecification is tend to lower the production by reinforcing a negative supply shock, and on the other hand, increases the inflation rate. As long as the central bank wants to be robust (so $\theta<\infty$ ), the policymaker will fear misspecification in this equation.

\subsection{Solving the worst-case model}

To find a closed-form solution for the robust control problem, we will look for the worstcase solution for the endogenous variables, $\pi_{t}$ and $y_{t}$, and the worst possible degree of misspecification or the evil agent's instrument, $v_{t}$. This equilibrium solution illustrates 
the central bank's worst fears of misspecification and therefore helps us to understand the design of the robust monetary policy.

We begin by looking for the optimal robust policy rule. The central bank sets its policy instrument $\pi_{t}$ in order to minimize the expected value of the loss function (12), taking $E_{t-1} \pi_{t}$ and $u_{t-1}$ as given, and after observing the current-period supply shock. This yields :

$$
\pi_{t}=\frac{\theta \xi(1-\gamma)}{\lambda(\theta-1)+\theta \xi^{2}(1-\gamma)^{2}}\left[\kappa+\xi(1-\gamma) E_{t-1} \pi_{t}-\xi\left(\phi u_{t-1}+\epsilon_{t}\right)\right]
$$

Then, assuming rational expectations for the private agents, we get :

$$
E_{t-1} \pi_{t}=\left(\frac{\theta}{\theta-1}\right) \frac{\xi}{\lambda}(1-\gamma)\left(\kappa-\xi \phi u_{t-1}\right) .
$$

The next step is to find the solutions of the worst-case model. Using equations (17), (18) and (10), we will thus find a solution for the endogenous variables $\pi_{t}$ and $y_{t}$, as:

$$
\begin{gathered}
\pi_{t}=\left(\frac{\theta}{\theta-1}\right) \frac{\xi}{\lambda}(1-\gamma)\left(\kappa-\xi \phi u_{t-1}\right)-\frac{\theta \xi^{2}(1-\gamma)}{\lambda(\theta-1)+\theta \xi^{2}(1-\gamma)^{2}} \epsilon_{t} . \\
y_{t}=\tilde{y}-\left(\frac{\theta}{\theta-1}\right) \kappa+\left(1+\frac{\xi \theta}{\theta-1}\right) \phi u_{t-1}+\left[1+\frac{\theta \lambda \xi}{\lambda(\theta-1)+\theta \xi^{2}(1-\gamma)^{2}}\right] \epsilon_{t} .
\end{gathered}
$$

and the worst possible degree of model misspecification will be given by

$$
v_{t}=-\left(\frac{1}{\theta-1}\right)\left(\kappa-\xi \phi u_{t-1}\right)+\frac{\lambda \xi}{\lambda(\theta-1)+\theta \xi^{2}(1-\gamma)^{2}} \epsilon_{t} .
$$

The above solution for the worst-case model is the reduced form under the worst possible case of misspecification. In this context, the evil agent chooses the specification errors to be as damaging as possible, and the optimal policy rule of the central bank and the expectations of the private sector reflect this misspecification. Equations (17) and (18) are both the reaction functions describing the central bank's policy rule and private agents' expectations of inflation. Both of them are dependent on the preference for robustness $(\theta)$ as the central bank fears misspecification in the model. Since the private sector shares the doubt of central bank about the reference model, it takes into account this uncertainty when forming their expectations. Equations (19) and (20) show that 
the equilibrium solutions for $\pi_{t}$ and $y_{t}$ depend not only on the central bank's preference for robustness $(\theta)$ and supply-side shocks of the current period, $\epsilon_{t}$, but also that of the last period, $u_{t-1}$. Equation (21) determines the worst possible degree of misspecification or the evil agent's instrument, $v_{t}$, which is restricted to respond to the same variables as the policymaker.

Using the above solution of the worst-case model, we next analyze how an increase in central bank preference for robustness (that is, a decrease in $\theta$ ) affects on the equilibrium the economy. We consider modest preference for robustness (so that, the worst-case model misspecification is not easily identified by the policymaker) and we analyze the effects of small decreases in $\theta$ starting from $\theta=\infty$. Thus to understand the effects of robustness on monetary policy we first study the worst-case model for inflation, output and inflation expectations. We establish the following propositions:

Proposition 1 In the worst-case model, a stronger preference for robustness of the central bank against misspecification (i.e., a decrease in $\theta$ ) increases the sensitivity of inflation $\left(\pi_{t}\right)$ and that of output $\left(y_{t}\right)$ to the supply-side shocks $\left(\epsilon_{t}\right)$.

Proof. Using equation (19) of the worst-case model to obtain the effects of a change in $\epsilon_{t}$ on the value of inflation $\pi_{t}$, we obtain:

$$
\frac{\partial \pi_{t}}{\partial \epsilon_{t}}=-\frac{\theta \xi^{2}(1-\gamma)}{\lambda(\theta-1)+\theta \xi^{2}(1-\gamma)^{2}} \quad \text { and } \quad \frac{\partial y_{t}}{\partial \epsilon_{t}}=\frac{\theta \lambda \xi}{\lambda(\theta-1)+\theta \xi^{2}(1-\gamma)^{2}}
$$

Then, the effect of a decrease in $\theta$ on the absolute value of the above derivative (22) is

$$
\begin{gathered}
-\frac{\partial\left|\frac{\partial \pi_{t}}{\partial \epsilon_{t}}\right|}{\partial \theta}=\frac{\lambda \xi^{2}(1-\gamma)}{\left[\lambda(\theta-1)+\theta \xi^{2}(1-\gamma)^{2}\right]^{2}}>0 \\
-\frac{\partial\left|\frac{\partial y_{t}}{\partial \epsilon_{t}}\right|}{\partial \theta}=\frac{\xi \lambda^{2}}{\left[\lambda(\theta-1)+\theta \xi^{2}(1-\gamma)^{2}\right]^{2}}>0
\end{gathered}
$$

As $\theta>1$ and $\gamma \in(0,1)$, we get $\partial \pi_{t} / \partial \epsilon_{t}<0, \partial y_{t} / \partial \epsilon_{t}<0$, and $-\partial\left|\frac{\partial \pi_{t}}{\partial \epsilon_{t}}\right| / \partial \theta<0$, $-\partial\left|\frac{\partial y_{t}}{\partial \epsilon_{t}}\right| / \partial \theta>0$.

Proposition 2 In the worst-case model, a stronger preference for robustness of the central bank against the model misspecification (i.e.,, a decrease in $\theta$ ) increases the 
expected inflation, increases the sensitivity of expected inflation to the inflation bias $(\kappa=\alpha \delta)$ due to the labour market distortions $(\delta)$, but decreases the sensitivity of expected inflation to the central bank's preference for inflation target $(\lambda)$.

Proof. To establish this proposition, we use (18) to derive the effects of a decrease in $\theta$ on the value of expected inflation as:

$$
-\frac{\partial\left(E_{t-1} \pi_{t}\right)}{\partial \theta}=\frac{\xi(1-\gamma)}{\lambda^{2}(\theta-1)}\left(\kappa-\xi \phi u_{t-1}\right)>0
$$

As $\theta>1$ and $\gamma \in(0,1)$, and assuming that $\kappa>\xi \phi u_{t-1}$, we obtain $-\partial\left(E_{t-1} \pi_{t}\right) / \partial \theta>$ 0 .Then, the effects of a decrease in $\theta$ on the absolute values of $\partial\left(E_{t-1} \pi_{t}\right) / \partial \lambda$ and $\partial\left(E_{t-1} \pi_{t}\right) / \partial \delta$ are given by

$$
\begin{gathered}
-\frac{\partial\left|\frac{\partial\left(E_{t-1} \pi_{t}\right)}{\partial \lambda}\right|}{\partial \theta}=-\frac{\xi(1-\gamma)}{\lambda^{2}(\theta-1)^{2}}\left(\kappa-\xi \phi u_{t-1}\right)<0 \\
-\frac{\partial\left|\frac{\partial\left(E_{t-1} \pi_{t}\right)}{\partial \delta}\right|}{\partial \theta}=\frac{\alpha \xi(1-\gamma)}{\lambda(\theta-1)^{2}}>0 . \\
-\frac{\partial\left|\frac{\partial\left(E_{t-1} \pi_{t}\right)}{\partial \phi}\right|}{\partial \theta}=-\frac{\xi^{2}(1-\gamma) u_{t-1}}{\lambda(\theta-1)^{2}}<0
\end{gathered}
$$

Note that in the above results a positive sign implies that the variable in question becomes more sensitive to that particular shock when robustness increases, and vice versa. A lower value of the parameter $\theta$ corresponds to a stronger fear of the central bank for model misspecification. In this context, the above results (23) and (24) show as the central bank has a stronger preference for robustness(a decrease in $\theta$ ), the higher the sensitivity of inflation to the supply-side shocks is. These results reveal that the robust central bank fears that inflation and output are more sensitives to shocks and therefore more volatile than in the reference model (see Leitemo and Soderstrom, 2008). In other words, the robust central bank fears that inflation is more volatile than in the reference model and responds more aggressively to the shock's effects on inflation, as the worst-case misspecification increases the volatility of all variables.

Equation (25) shows that a fear of model misspecification leads to a higher expected inflation. As the less central bank considers that its reference model is robust (so a 
stronger preference for robustness) and since the private sector shares the doubt of central bank about the reference model, it takes into account this doubt when forming their expectations of inflation. On the other hand, (26) shows that as the less central bank considers that its reference model is robust (a stronger preference for robustness), the lower the sensitivity of expected inflation to the central bank's preference for the inflation target (or aversion for inflation) is. That is, the robust central bank fears that expected inflation is more volatile than in the reference model and responds more aggressively to the variations on the weight assigned to the inflation target. However, a crucial role in this context plays the persistence of the supply-side shocks generating an inflation persistence which will be affected as well by the central bank's robustness.

\section{$3 \quad$ Monetary policy and inflation persistence}

The main focus of our next analysis concerns the relationship between of the central bank's preference for robustness and the inflation persistence as well as the resulting output cost of disinflation.

\subsection{Introducing inflation persistence}

We attempt here to determine the relationship between the parameter of preference for robustness $\theta$ and the degree of inflation persistence. The inflation persistence, captured by the relation : $\pi_{t}=\rho_{\pi} \pi_{t-1}$, may be calculated by using the correlation coefficient $\rho_{\pi}$ between $\pi_{t}$ and $\pi_{t-1}$, as :

$$
\rho_{\pi}=\operatorname{Cov}\left(\pi_{t}, \pi_{t-1}\right) / \operatorname{Var}\left(\pi_{t}\right)
$$

To determine $\operatorname{Var}\left(\pi_{t}\right) \equiv E\left[\left(\pi_{t}-\bar{\pi}\right)^{2}\right]$ and $\operatorname{Cov}\left(\pi_{t}, \pi_{t-1}\right) \equiv E\left[\left(\pi_{t}-\bar{\pi}\right)\left(\pi_{t-1}-\bar{\pi}\right)\right]$, the unconditional mean of the inflation $\bar{\pi}$ can be written as :

$$
\bar{\pi}=\frac{\theta}{\lambda(\theta-1)} \xi(1-\gamma) \kappa .
$$

Due to the overly ambitious output target of the policymaker, $\kappa$, the unconditional 
inflation is not zero, but with a bias depending on the degree of model uncertainty. Combining equations (19) and (30), the difference of inflation rate from its mean is

$$
\pi_{t}-\bar{\pi}=-\theta \xi^{2}(1-\gamma)\left[\frac{\phi}{\lambda(\theta-1)} u_{t-1}+\frac{1}{\lambda(\theta-1)+\theta \xi^{2}(1-\gamma)^{2}} \varepsilon_{t}\right] .
$$

and the unconditional variance of inflation is of the form

$$
\operatorname{Var}\left(\pi_{t}\right)=\left[\frac{\theta \xi^{2}(1-\gamma)}{\lambda(\theta-1)}\right]^{2} \frac{\left\{\phi^{2}\left[\lambda(\theta-1)+\theta \xi^{2}(1-\gamma)^{2}\right]^{2}+\lambda^{2}(\theta-1)^{2}\right\} \sigma_{u}^{2}}{\left[\lambda(\theta-1)+\theta \xi^{2}(1-\gamma)^{2}\right]^{2}}
$$

The variability of the inflation is determined principally by the variance of the supply shock $\sigma_{u}^{2}$ premultiplied by a coefficient depending upon the structural and preference parameters.

To obtain the covariance of the inflation rates $\pi_{t}$ and $\pi_{t-1}$, we use equation (19) for the period $t-1$ and, from equation (8), the fact that $\phi u_{t-2}=u_{t-1}-\epsilon_{t-1}$. Finally, considering that the unconditional mean of $\pi_{t-1}$ is also equal to $\bar{\pi}$, and assuming that $E\left(\epsilon_{t} \epsilon_{t-1}\right)=E\left(\epsilon_{t-1} u_{t-1}\right)=E\left(\epsilon_{t} u_{t-1}\right)=0$, we obtain:

$$
\operatorname{Cov}\left(\pi_{t}, \pi_{t-1}\right)=\left[\frac{\theta \xi(1-\gamma) \xi}{\lambda(\theta-1)}\right]^{2} \phi \sigma_{u}^{2}
$$

Finally, from equations (29), (32) and (33) follows the correlation coefficient (or inflation persistence), $\rho_{\pi}$, between $\pi_{t}$ and $\pi_{t-1}$, as:

$$
\rho_{\pi}=\frac{\phi \Omega^{2}}{\phi^{2} \Omega^{2}+\lambda^{2}(\theta-1)^{2}}
$$

where $\Omega=\lambda(\theta-1)+\theta \xi^{2}(1-\gamma)^{2}>0$. It is easy to verify that the correlation coefficient satisfies $\rho_{\pi} \geq 0$. For insuring that also $\rho_{\pi} \leq 1$, we need : $(\theta-1)^{2} \geq \phi \Omega^{2} /\left[\lambda^{2}(1+\phi)\right]$. Solving this inequality yields

$$
\theta \geq(1-\sqrt{\phi /(1-\phi)})\left\{1-\sqrt{\phi /(1-\phi)}\left[1+\xi^{2}(1-\gamma)^{2} / \lambda\right]\right\}^{-1}
$$

This condition determine another lower bound of the central bank's preference for robustness $\theta$ which depends on the structure parameters $(\xi, \gamma, \phi)$ and the relative weight assigned to inflation stabilization $\lambda$. Therefore, the degree of inflation persistence $\rho_{\pi}$ 
is determined by the central bank's preference parameters (i.e. preference for inflation stabilization $\lambda$, and the preference for robustness $\theta)$ and the structural parameters $(\gamma$, $\xi$ and $\phi)$.

\subsection{The effects of central bank robustness}

We discuss in this section the effects of central bank's preference for robustness on the degree of inflation persistence and the sacrifice ratio. In this respect, we establish the following propositions:

Proposition 3 The increase in the preference for robustness of the central bank against the model misspecification (i.e., a decrease in $\theta$ ) increases the inflation persistence $\left(\rho_{\pi}\right)$

Proof. To establish this result, we take the first derivative of $\rho_{\pi}$ with respect to $\theta$ from equation (34). This yields

$$
\frac{\partial \rho_{\pi}}{\partial \theta}=-\frac{2 \phi\left[\lambda(\theta-1)+\theta \xi^{2}(1-\gamma)^{2}\right]^{2} \lambda^{2}(\theta-1)}{\left\{\phi^{2}\left[\lambda(\theta-1)+\theta \xi^{2}(1-\gamma)^{2}\right]^{2}+\lambda^{2}(\theta-1)^{2}\right\}^{2}}
$$

As $\theta>1$ and $\gamma \in(0,1)$, from the above derivative (36), we obtain $\frac{\partial \rho_{\pi}}{\partial \theta}<0$.

The intuition behind the above result (36) is that as the central bank has a stronger preference for robustness (i.e., a decrease in $\theta$ ), or as the less central bank believes that its reference model is robust, the higher the inflation persistence will be. In this case, according to our previous the result (25) in proposition 2, a fear of model misspecification leads to a higher expected inflation and thus a greater inflation persistence. This inflation persistence may arise for several reasons: the inertia that slowly adjusting inflation expectations, the inertia of imperfect credibility due to a low degree of central bank's aversion for inflation or degree of central bank independence (designed by the parameter $\lambda$ ), a higher degree of shock persistence when the supply-side shocks are highly correlated (designed by the parameter $\phi$ ), or the inertia that wage and price 
contracts captured here by a low degree of wage indexation (parameter $\gamma$ ). In this context, the following propositions can be established.

Proposition 4 A stronger preference for robustness against the model misspecification (i.e., a decrease in $\theta$ ) decreases the sensitivity of inflation persistence $\left(\rho_{\pi}\right)$ to the central bank's aversion of inflation $(\lambda)$.

Proof. To set-up this result, we take from equation (34) the first derivative of $\rho_{\pi}$ with respect to $\lambda$ and we get

$$
\frac{\partial \rho_{\pi}}{\partial \lambda}=-\frac{2 \phi \theta A^{2}\left[\lambda(\theta-1)+\theta A^{2}\right] \lambda(\theta-1)^{2}}{\left[\lambda^{2}(\theta-1)^{2}\left(1+\phi^{2}\right)+\phi^{2} \theta^{2} A^{4}+2 \lambda \phi^{2} \theta A^{2}(\theta-1)\right]^{2}}<0
$$

As $\theta>1$ and $A \equiv \xi(1-\gamma)>0$ since $\gamma \in(0,1)$, we obtain : $\partial \rho_{\pi} / \partial \lambda<0$.Then, the effect of a decrease in $\theta$ on the value of the above derivative (37) is given by

$$
\begin{aligned}
-\frac{\partial\left|\frac{\partial \rho_{\pi}}{\partial \lambda}\right|}{\partial \theta}= & -\frac{2 \phi \lambda A^{2}(\theta-1)\left\{\lambda^{3}(\theta-1)^{3}\left(1+\phi^{2}\right)+2 \theta \lambda^{2} A^{2}(\theta-1)^{2}\right\}}{\left[\lambda^{2}(\theta-1)^{2}\left(1+\phi^{2}\right)+\phi^{2} \theta^{2} A^{4}+2 \lambda \phi^{2} \theta A^{2}(\theta-1)\right]^{3}} \\
& -\frac{2 \phi \lambda A^{2}(\theta-1)\left\{\phi^{2} \theta^{2} A^{4}\left[2 \theta A^{2}+3 \lambda(\theta-1)\right]\right\}}{\left[\lambda^{2}(\theta-1)^{2}\left(1+\phi^{2}\right)+\phi^{2} \theta^{2} A^{4}+2 \lambda \phi^{2} \theta A^{2}(\theta-1)\right]^{3}}
\end{aligned}
$$

As $\theta>1$ and $A \equiv \xi(1-\gamma)>0$ since $\gamma \in(0,1)$, we obtain : $-\partial\left|\frac{\partial \rho_{\pi}}{\partial \lambda}\right| / \partial \theta<0$.

Proposition 5 An increase in the preference for robustness of the central bank against the model misspecification (i.e., a decrease in $\theta$ ) decreases the sensitivity of inflation persistence $\left(\rho_{\pi}\right)$ to the degree of supply-side shock persistence $(\phi)$.

Proof. To set-up this result, we take from equation (34) the first derivative of $\rho_{\pi}$ with respect to $\phi$ and we obtain

$$
\frac{\partial \rho_{\pi}}{\partial \phi}=\frac{\left[\lambda^{2}(\theta-1)^{2}\left(1-\phi^{2}\right)-\theta^{2} \xi^{4}(1-\gamma)^{2}\right] \Omega^{2}}{\left\{\phi^{2}\left[\lambda(\theta-1)+\theta \xi^{2}(1-\gamma)^{2}\right]^{2}+\lambda^{2}(\theta-1)^{2}\right\}^{2}},
$$

Assuming that $\lambda^{2}(\theta-1)^{2}\left(1-\phi^{2}\right)>\theta^{2} \xi^{4}(1-\gamma)^{2}$ (for example, when $\gamma \rightarrow 1$ ), we obtain: $\frac{\partial \rho_{\pi}}{\partial \phi}>0$. Then, the effect of a decrease in $\theta$ on the value of the above derivative is 
given by

$$
-\frac{\partial\left|\frac{\partial \rho_{\pi}}{\partial \phi}\right|}{\partial \theta}=-\frac{2 \lambda^{2}(\theta-1)\left[\theta^{2} \xi^{4}(1-\gamma)^{4}+\lambda^{2}(\theta-1)^{2}\left(3 \phi^{2}-1\right)\right] \Omega^{2}}{\left\{\phi^{2}\left[\lambda(\theta-1)+\theta \xi^{2}(1-\gamma)^{2}\right]^{2}+\lambda^{2}(\theta-1)^{2}\right\}^{3}}<0
$$

As $\theta>1$ and $\gamma \in(0,1)$, when $3 \phi^{2}>1 \Rightarrow \phi>\sqrt{1 / 3}$, we obtain: $-\partial\left|\frac{\partial \rho_{\pi}}{\partial \phi}\right| / \partial \theta<0$.

Proposition 6 The increase in the preference for robustness of the central bank (i.e., a decrease in $\theta)$ decreases the sensitivity of inflation persistence $\left(\rho_{\pi}\right)$ to the wage indexation parameter $(\gamma)$.

Proof. To establish this result, we recall that the equation (34) may be transformed as

$$
\rho_{\pi}=\left(\phi+\frac{\lambda^{2}}{\phi} g(\theta)\right)^{-1} \text { with } g(\theta)=\left[\frac{(\theta-1)}{\lambda(\theta-1)+\theta \xi^{2}(1-\gamma)^{2}}\right]^{2}
$$

Then, we get respectively the first derivative of $\rho_{\pi}$ with respect to $g$ and the first derivative of $g$ with respect to $\gamma$ as

$$
\frac{\partial \rho_{\pi}}{\partial g}=-\frac{\lambda^{2} \phi}{\left[\phi+\lambda^{2} g\right]^{2}}<0, \frac{\partial g}{\partial \gamma}=\frac{4 \theta \xi^{2}(1-\gamma)(\theta-1)^{2}}{\left[\lambda(\theta-1)+\theta \xi^{2}(1-\gamma)^{2}\right]^{3}}>0
$$

Using the above results we get :

$$
\frac{\partial \rho_{\pi}}{\partial \gamma}=\frac{\partial \rho}{\partial g} \cdot \frac{\partial g}{\partial \gamma}<0
$$

Finally, the effect of a decrease in $\theta$ on the value of the sensitivity of inflation persistence to wage indexation $\left(\partial \rho_{\pi} / \partial \gamma\right)$ is given by

$$
-\frac{\partial\left|\frac{\partial \rho_{\pi}}{\partial \gamma}\right|}{\partial \theta}=-\frac{4 \theta \xi^{2}(1-\gamma)(\theta-1)\left[2 \theta \xi^{2}(1-\gamma)^{2}+\lambda(\theta-1)\right]}{\left[\lambda(\theta-1)+\theta \xi^{2}(1-\gamma)^{2}\right]^{4}}<0
$$

As $\theta>1$ and $\gamma \in(0,1)$, for the above derivative we obtain : $-\partial\left|\frac{\partial \rho_{\pi}}{\partial \gamma}\right| / \partial \theta<0$.

The intuition behind these results is the following. According to results (37), (39) and (42) respectively in the three above propositions, the inflation persistence may arise from several reasons, including: i) the inertia that imperfect credibility (due to a low degree of central bank independence) may impart to inflation, ii) the inertia that 
supply-side productivity shocks may impart to inflation, or iii) the inertia that nominal wage indexation impart to inflation. The first result reveals a negative relationship between the relative weight assigned by the central bank on inflation stabilization (or central bank's aversion to inflation, $\lambda$ ) and the inflation persistence. The second result illustrates a positive relationship between the inertia of supply-side productivity shocks and the inflation persistence. In other words, a lower degree of the inertia of supply-side productivity shocks (i.e. a lower $\phi$ ) leads to a lower degree of the inflation persistence. The third result shows a negative relationship between the degree of wage indexation (parameter $\gamma$ ) and the inflation persistence or a positive relationship between the inertia of wage and price contracts and the inflation persistence.

Further, we show respectively in (38), (40) and (43) that the above three results on inflation persistence are deteriorated when as the less central bank believes that its reference model is robust. That is, as the central bank has a stronger preference for robustness (i.e., a decrease in $\theta$ ), the lower the sensitivity of the above three effects on inflation persistence will be. These results confirm the outcome of proposition 3 , so that an increase of central bank's preference for robustness, increase inflation persistence.

\subsection{Speed and cost of disinflation}

We examine now the effects of central bank robustness on the output cost disinflation or the sacrifice ratio through its effects on the inflation persistence (or speed of disinflation). The sacrifice ratio is computed here by first adding up all output losses incurred during the phase of disinflation and then dividing this sum by the achieved reduction of inflation. The following proposition can be established.

Proposition 7 The increase in the preference for robustness of the central bank (i.e., a decrease in $\theta$ ), by increasing the degree of inflation persistence (closely connected to the speed of disinflation), increases the output cost of disinflation or sacrifice ratio.

Proof. To establish this result, we consider the sacrifice ratio whose definition is

$$
s r=-\sum_{i=1}^{\infty}\left(\bar{y}-y_{t+i}\right) / \Delta \pi,
$$


We compute as the size of the disinflation $\Delta \pi$ between $t$ and $t+1: \Delta \pi=\pi_{t+1}-\pi_{t}$. Starting from a situation with an initial inflation $\pi_{t}$ equal to its mean $\bar{\pi}$ and assuming that the inflation rate follows an $\operatorname{AR}(1)$ process: $\pi_{t+1}=\rho_{\pi} \pi_{t}=\rho_{\pi} \bar{\pi}$, we can write:

$$
\Delta \pi=-\left(1-\rho_{\pi}\right) \bar{\pi}
$$

where it is assumed that the average inflation rate is considered as given when policymaker determines the inflation rate in a specific period. Using respectively equations equation (44) and equation (45), we obtain:

$$
\partial(s r) / \partial \rho_{\pi}=\sum\left(\bar{y}-y_{t+i}\right) /\left(\rho_{\pi}-1\right)^{2} \bar{\pi}>0
$$

Combining the above result with the Proposition 3 yields :

$$
\frac{\partial(s r)}{\partial \theta}=\frac{\partial(s r)}{\partial \rho_{\pi}} \cdot \frac{\partial \rho_{\pi}}{\partial \theta}<0
$$

As $\partial \rho_{\pi} / \partial \theta<0$ from (36) and $\partial(s r) / \partial \rho_{\pi}>0$ from (46), we get : $\partial(s r) / \partial \theta>0$.

According to this result, the model predicts a cost of disinflation which is increasing with the degree of the central bank preference for robustness. In particular, the intuition behind this results is that a higher preference for robustness of central bank is positively associated with the inflation persistence and thus negatively with the speed of disinflation. Therefore, this paper shows that the output cost of disinflation is higher when the less the central bank believes that its reference model is robust and thus its preference for robustness is higher.

\subsection{A numerical example}

To demonstrate the analytical results more intuitively, we provide following numerical simulations. All parameters values are presented in table (1).

We computes first the degree of inflation persistence as a function of the degree of model uncertainty . ${ }^{1}$ Figure 1 shows how the degree of inflation persistence varies

\footnotetext{
${ }^{1}$ justify the choice of parameter values.
} 
Table 1: Parameter values for the model calibration

\begin{tabular}{|c|c|c|c|c|c|c|}
\hline \multicolumn{3}{|c|}{ Model } & \multicolumn{2}{|c|}{ Shocks } & \multicolumn{2}{|c|}{ Policy } \\
\hline$\phi$ & $\xi$ & $\gamma$ & $\varepsilon_{t}$ & $u_{t-1}$ & $\lambda$ & $k$ \\
\hline 0.77 & 1.5 & 0.5 & 0.001 & 0.01 & 2 & 1 \\
\hline
\end{tabular}

with different preference for robustness. ${ }^{2}$ The decreasing curve verifies the negative relationship between $\rho$ and $\theta$. As the degree of model uncertainty increases (smaller values of $\theta$ ), the inflation persistence increases. Moreover, when $\theta$ takes small values, a trivial decrease induces a rather large increase in the degree of inflation persistence. Whereas, as the values of $\theta$ are higher, the inflation persistences are less sensitive to its variation. As shown in figure 1 , the curve become more and more flat, as the values of $\theta$ increase. This observation yields an interesting implication. If the private agents and central bank, both believes rather in their reference model(relative great values of $\theta$ ), a small increase of preference for robustness does not affect significantly the inflation persistence. While their doubts about the reference model turn out to be severer (relative small values of $\theta$ ), the inflation persistences become more sensitive to changes in preference for robustness.

Another factor that influences the process of inflation dynamics is the exogenous wage indexing parameter $\gamma$. Lawler(2000) shows that the wage setting behavior has important implications to monetary institution design. It is of interest to examine the effect of wage indexing behavior on the disinflation rate. For this purpose, we repeat the simulation with the same numerical value for $\lambda, \phi, \xi$ but make the indexing parameter vary. Figure 2 shows the evolutions of degree of inflation persistence under different indexing parameters, taking account of model uncertainty. Under the same degree of model uncertainty, inflations exhibit a higher inertia if the wage indexing rate is relatively low. According to the optimal conditions (14) and (16), a small value of wage indexing worsens the output-inflation trade-off (i.e. $1-\gamma$ becomes greater ) and strengthens the misspecification. Consequently, inflation become more sluggish under a low indexing wage setting behavior.

\footnotetext{
${ }^{2}$ When choosing the numerical values for $\theta$, we have verified that the lower bound condition (35) are satisfied under this parameter set.
} 


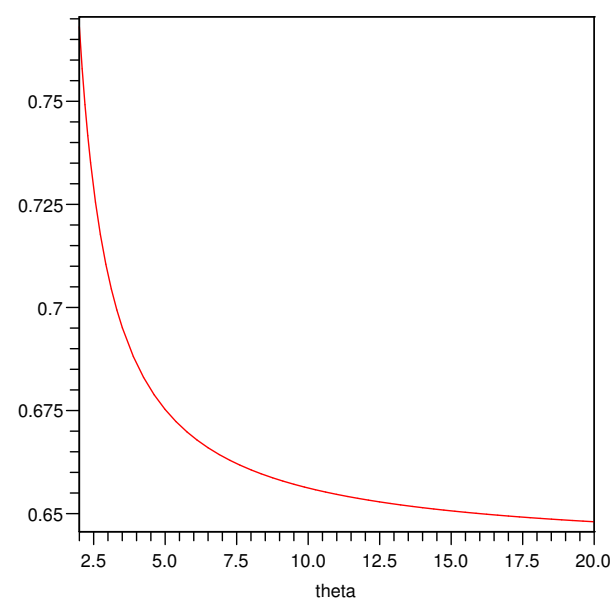

Figure 1: Inflation persistence under model uncertainty, (the impact of $\theta$ )

Using the expression of output $y_{t}$ given by Eq.(20), we can obtain

$$
\bar{y}-y_{t+i}=\frac{1}{\theta-1} k-\left(1+\frac{\xi \theta}{\theta-1}\right) \phi u_{t-1}-\left[1+\frac{\theta \lambda \xi}{\lambda(\theta-1)+\theta A^{2}}\right] \varepsilon_{t} .
$$

Inserting it into Eq. (44), together with Eq.(30) and (45), we can compute the ratio sacrifice. Figure 3 plots the changes in ratio sacrifice as the preference for robustness decreases (an increase in $\theta$ ) with the parameter values given in table 1 . The decreasing curve is in accordance with the negative relationship between $s r$ and $\theta$ shown by Eq. $(47)$.

\section{Concluding remarks}

This paper examines the relationship between the preference for robustness of central bank against the model misspecification and the inflation persistence or the speed of disinflation. We use a simple monetary game model in which a stroger preference for robustness of central bank is positively associated with the inflation persistence and therefore negatively related with the speed of disinflation. In this context, we show that the output cost of disinflation (or sacrifice ratio), associated positively with the inflation persistence (or the speed of disinflation), will be higher when the preference for robustness is higher and thus less the central bank believes that its reference model 


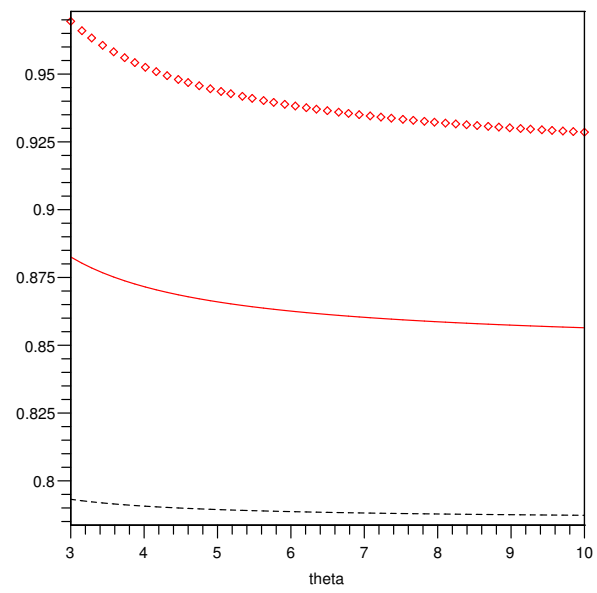

Figure 2: Inflation persistence under model uncertainty, with different indexing parameters

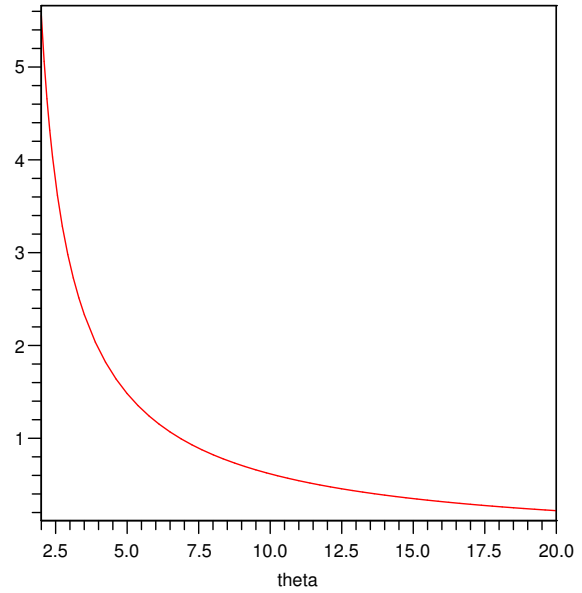

Figure 3: Sacrifice ratio under model uncertainty 
is robust.

The policy implication lurking behind this finding is that a cautious central bank is well-advised to design and implement its monetary policy under the assumption that inflation persistence is high when there is considerable uncertainty about the misspecification of the reference model. Our results show that robust monetary policy is tilted towards the specification that induces a high degree of inflation persistence. Specifically, we show that robust monetary policy is characterised by a relatively aggressive response to inflation and exhibit a substantial degree of inflation inertia and the speed of disinflation. 


\section{References}

[1] Alesina, A., and Tabellini, G., (1987), "Rules and Discretion with Noncoordinated Monetary and Fiscal Policies", Economic Inquiry, 25, 619-630.

[2] Angeloni, I., Coenen, G., Smets, F., 2003. Persistence, the transmission mechanism and robust monetary policy. Scottish Journal of Political Economy 50, 527-549.

[3] Calvo, G.A., 1983. Staggered prices in a utility-maximizing framework. Journal of Monetary Economics 12, 383-398.

[4] Christiano, L.J., Eichenbaum, M., Evans, C., 2005. Nominal rigidities and the dynamic effects of a shock to monetary policy. Journal of Political Economy 113, $1-45$.

[5] Cogley, T., Sargent, T.J., 2001. Evolving post-world war II US inflation dynamics. In: Bernanke, B.S., Rotemberg, J.J. (Eds.), NBER Macroeconomics Annual, vol. 16. MIT Press, Cambridge, MA.

[6] Fuhrer, J.C., Moore, G.R., 1995. Inflation persistence. Quarterly Journal of Economics 110, 127-160.

[7] Taylor, J.B. (2000), "Low inflation, pass-through, and the pricing power of firms". European Economic Review 44, 1389-1408.

[8] Barro, R. and Gordon, D. (1983) A Positive Theory of Monetary Policy in a Natural Rate Model. Journal of Political Economy, 91, 589-610.

[9] Cukierman, A., (2001) "Accountability, Credibility, Transparency and Stabilization Policy in the Eurosystem ", in C. Wyplosz (ed.), The Impact of EMU on Europe and the Developing Countries Oxford University Press, 40-75.

[10] Hansen, L. P. and Sargent, T. J., (2003) Robust Control for Forward Looking Models, Journal of Monetary Economics, 50(3), 586-604.

[11] Hansen, L. P. and Sargent, T. J., (2004) Robust Control and Model Uncertainty in Macroeconomics, Princeton University Press. 
[12] Cukierman, A., (2005) "The Limits of Transparency" Working Paper, Presented at the session on "Monetary Policy Transparency and Effectiveness" at the January 2006 Meeting of the American Economic Association.

[13] Demertzis, Maria, A. Hughes Hallet (2003) "Central Bank Transparency in Theory and Practice", DNB Staff Reports 2003, No. 105, De Nederlandsche Bank.

[14] Geraats, Petra M., (2000). "Why Adopt Transparency? The Publication of Central Bank Forecasts." CEPR Discussion Paper 2582, October.

[15] Geraats, Petra M.,(2002). "Central Bank Transparency.” Economic Journal 112, F532-F565, November.

[16] Kilponen, J., 2003, A Positive Theory of Monetary Policy and Robust Control, Bank of Finland Discussion Papers 18.

[17] Kydland, F. and Prescott E. (1977) Rules Rather than Discretion: the Inconsistency of Optimal Plans. Journal of Political Economy, 85, 473-491.

[18] Leitemo, K and Söderström, U (2004) Robust monetary policy in a small open economy. Manuscript, Norwegian School of Management andUniversità Bocconi.

[19] Rogoff, K., 1985, The Optimal Degree of Commitment to an Intermediate Monetary Target. Quarterly Journal of Eco-nomics, 100, 1169-1189.

[20] Giordani, P and Söderlind, P (2004) Solution of macromodels withHansen-Sargent robust policies: Some extensions. Journal of Economic Dynamics and Control, 28 (12), $2367-2397$.

[21] Onatski, A and Williams, N (2003) Modeling model uncertainty. Journal of the European Economic Association, 1 (5), 1087-1122.

[22] Walsh, C (2004) Robust optimal instrument rules and robust control: An equivalence result. Manuscript, University of California, Santa Cruz. 